\title{
A hexapole magnetic guide for neutral atomic beams
}

\author{
J.P. Beardmore, A.J. Palmer, K.C. Kuiper, ${ }^{*}$ and R.T. Sang ${ }^{\dagger}$ \\ Centre for Quantum Dynamics, Griffith University, Nathan, QLD 4111 Australia
}

(Dated: June 3, 2009)

\begin{abstract}
In this paper we present a multiple element magnetic device to guide atoms using a spatially inhomogeneous magnetic field formed by a series of permanent hexapole magnets. The operation of the device is demonstrated using an enhanced beam of neon atoms in the ${ }^{3} P_{2}$ metastable state. These atoms are guided around a bend of 30 degrees from their original path. A flux of $4.35 \times 10^{9} \pm$ $2 \times 10^{7}$ atoms s $^{-1}$ was measured after the device yielding a transmission efficiency of approximately $9 \%$ of the input flux. Simulations of the center of mass motion of the atoms through the magnetic guide have been performed giving reasonable agreement with the experimental results.
\end{abstract}

PACS numbers: $37.10 .-\mathrm{x}, 37.20 .+\mathrm{j}$

*Department of Applied Physics, Eindhoven University of Technology, PO Box 513, 5600 MB Eindhoven, The Netherlands

${ }^{\dagger}$ Electronic address: r.sang@griffith.edu.au 


\section{INTRODUCTION}

The application of static electromagnetic elements to manipulate atomic beams can be traced back to the 1920s when Stern and Gerlach performed their famous experiments demonstrating the intrinsic angular momentum of electrons via the deflection of silver atoms passing though an inhomogeneous magnetic field [1]. Friedburg and Paul advanced the manipulation of atomic beams by using a hexapole magnet to create a spatially varying magnetic field to focus atoms from a thermal atomic beam [2]. More recently there has been a significant resurgence in the enhancement of neutral atomic beams to produce slow, "bright" continuous atomic beams via the application of electromagnetic fields. This enhancement or modification of atomic beams has spawned the research field of atom optics $[3,4]$. Enhanced atomic beams have been used for a diverse range of experiments for example, the loading of atom traps, atom interferometers, as well as their application in surface science [3]. Enhanced atomic beams have also been used to create nanostructures in a process called nanolithography. This process involves the manipulation of atomic beams to create nanoscale structures on surfaces [5]. This has been accomplished using two generic techniques; the first technique directly deposits atom to an appropriately prepared surface and is termed direct deposition. The second technique relies on the exposure of a surface, covered with a specific resist, to energetic atoms. Patterns are then transferred to the surface after the application of an etching technique. The second technique has mainly utilized metastable atomic beams formed by gas discharge sources. Noble gas atoms in a metastable state exhibit internal energies in the range of $8-20 \mathrm{eV}$, which is sufficient to damage, upon collision, a resist layer formed from a self-assembled monolayer (SAM) of alkanethiols. The areas damaged by exposure to the metastable rare gas atoms can then be transferred into the underlying layers via subsequent chemical etching processes [6]. By modulating the atomic beam incident upon the surface, via either a physical or near resonant standing wave light mask, patterning of the sample can be achieved. To achieve minimum feature sizes on the sample, the atomic beam must be cold, monochromatic, and consist mainly of atoms in the metastable state.

The conventional method of producing metastable atomic beams is through gas discharge systems [7]. However, it is an inefficient process with only $\sim 0.01 \%$ of the gas atoms emerging from the discharge being excited to the metastable state. The resulting atomic 
beam is therefore a combination of metastable and ground state atoms and also contains electrons, ions, and UV photons, all of which may lead to undesirable lithographic patterning. The contribution of the UV photons to the final lithographic pattern is still undetermined, though their ability to damage the resist layer has been observed by performing quenching experiments with our system. Charged particles are removed from the atomic beam by the application of an appropriate transverse electric field [8], deflecting these components from the experimental axis. The removal of UV photons from the beam requires a more complicated experimental arrangement in order to deflect the metastable gas atoms from the path of the photons. Current techniques available to separate the metastable atoms from the UV photons include; transverse deflection via radiation pressure [9], deflection utilizing a two-dimensional magneto-optical molasses (2D-MOM) [10], and the focusing of the metastable atoms around a physical beam block [11]. The first two approaches deflect the metastable atoms from the experimental axis in order to separate the components to the atomic beam, while the third approach utilizes a physical barrier to introduce a shadow into the imaging plane.

The motivation behind this work is to produce a UV free metastable atomic beam for application in atom lithography. The work presented here demonstrates a novel approach to the guiding of metastable neon atoms using a series of hexapole magnetic lens elements and is capable of producing a UV free atomic beam. This approach is not limited to metastable atoms but can be used to guide any atomic species with a magnetic moment. It could also be beneficial for the loading of magneto-optical traps that are operated in UHV environments as the apparatus that we present here could be simultaneously be used as a conductance aperture for a differential pumping stage. Since the nature of the device we present here deflects atoms from the original path from that of the source, increases in vacuum pressure due to unslowed or undesirable constituents are removed.

\section{EXPERIMENTAL APPARATUS}

A schematic of the atomic beam apparatus used in this investigation is provided in Figure 1. A beam of neon atoms in both the ${ }^{3} \mathrm{P}_{0}$ and ${ }^{3} \mathrm{P}_{2}$ metastable states (which are designated by $\mathrm{Ne}^{*}$ ) is generated in a glow DC discharge source with the approximate proportions of $1 / 6$ and $5 / 6$ respectively $[12-14] \equiv$ The discharge is struck between a cathode pin and the 
external skimmer plate, while the atoms expand supersonically through a liquid nitrogen cooled nozzle constructed from boron nitride. The atomic beam then passes through a $1 \mathrm{~mm}$ aperture in the skimmer, into the differentially pumped optical collimation region. During operation, the source region, which is pumped by an oil diffusion pump, maintains a pressure of $8 \times 10^{-5}$ Torr, while the collimation region remains an order of magnitude lower at $1 \times 10^{-6}$ Torr. The emergent metastable neon beam has a most probable velocity of $580 \pm 20 \mathrm{~m} \mathrm{~s}^{-1}$, as measured by time-of-flight methods. A set of orthogonal, nearly parallel mirrors [15], located $50 \mathrm{~mm}$ downstream reduces the divergence of the atomic beam to $6.5 \pm 2.6 \mathrm{mrad}$ via transverse laser cooling by multiple reflections of $640.4 \mathrm{~nm}$ laser radiation generated by a Coherent 899 ring dye laser. This wavelength corresponds to the ${ }^{3} P_{2} \rightarrow{ }^{3} D_{3}$ closed optical transition and is the laser cooling transition for $\mathrm{Ne}^{*}[16]$. Charged constituents of the beam are also removed in this region using electrostatic deflector plates, which apply a transverse electric field of approximately $60 \mathrm{kV} \mathrm{m}^{-1}$.

The total atom flux is measured using a $25 \mathrm{~mm}$ diameter, biased, chemically cleaned, stainless steel Faraday plate. The $16.6 \mathrm{eV}$ internal energy of $\mathrm{Ne}^{*}$ [17] is sufficient to eject secondary electrons upon collision with the metal surface. The flux measured immediately after the electrostatic deflector plates was increased from $6.7 \times 10^{11} \pm 1 \times 10^{10}$ atoms s $^{-1}$ to $1.60 \times 10^{12} \pm 4 \times 10^{10}$ atoms $^{-1}$ by introduction of the optical collimation. These values were calculated using a secondary electron ejection coefficient of 0.3 for a chemically cleaned stainless steel plate [18]. Quenching experiments using a nitrogen buffer gas indicated that the UV photons account for $6 \%$ of the measured Faraday plate current [8].

The next stage of atomic beam preparation involves the longitudinal cooling and decelerating of the metastable neon atoms. This is achieved via a Zeeman-slower constructed as a tapered solenoid with a maximum field strength of $0.75 \mathrm{~T}$ which is $920 \mathrm{~mm}$ in length. Dual $20 \mathrm{~mm}$ laser beams incident at $15 \mathrm{mrad}$, relative to the solenoid axis, cross over the entire length of the Zeeman-slower and are tuned to $-140 \mathrm{MHz}$ from the resonant, laser cooling frequency. This is a new slowing beam arrangement and allows for a relatively large (8 $\mathrm{mm}$ ) aperture to be obtained at the input mirror facet. A recollimation optical molasses immediately after the Zeeman-slower solenoid ensures maximum flux is achieved through the aperture, $8.5 \times 10^{10} \pm 1 \times 10^{9}$ atoms $^{-1}$. This flux is then incident on the $23.5 \mathrm{~mm}$ aperture at the opening to the multiple magnetic hexapole element atomic beam guide. 


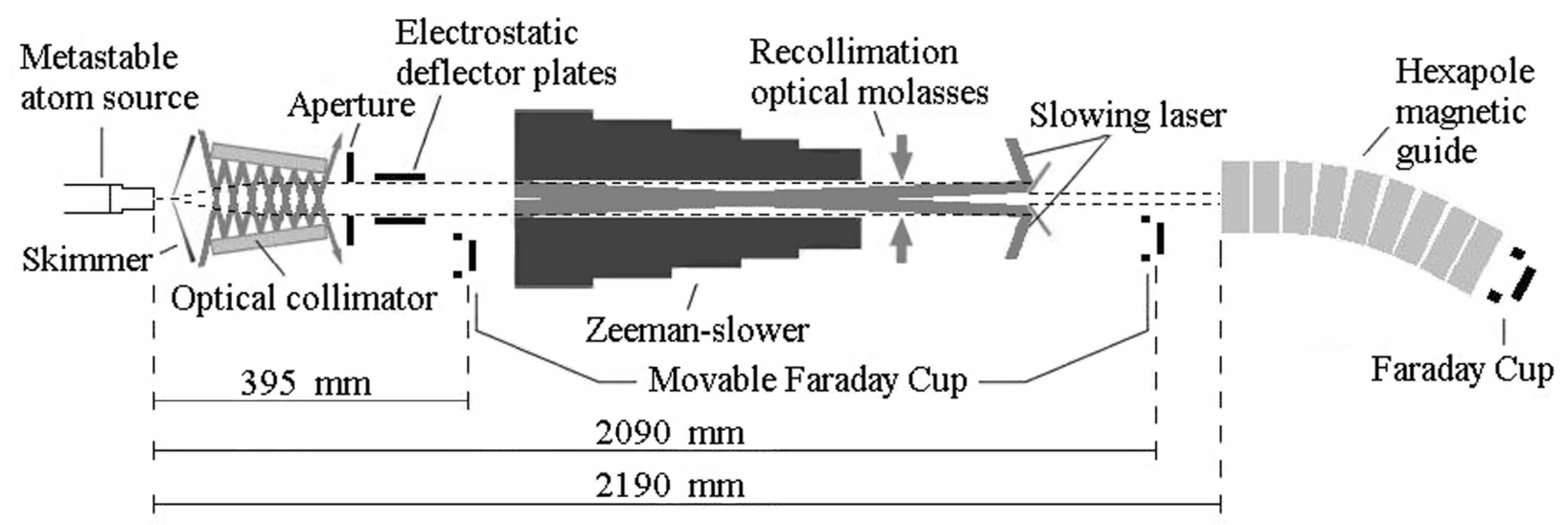

FIG. 1: Schematic of the metastable atomic beamline. (Not to scale).

\section{MAGNETIC GUIDE DESIGN AND MODELLING}

Similar devices utilizing quadrupole and octupole magnetic fields to guide alkali metal atoms have previously been demonstrated $[19,20]$. While larger field gradients are achievable using an octupole arrangement, the atomic beam is confined to a larger proportion of the central aperture of the guide, reducing the emergent atomic flux density. Unlike the other magnetic guides the application of this device utilizes hexapole magnetic fields which have been chosen as the guiding lens elements since the force exerted on atoms as they traverse each element increases linearly with respect to distance from the axis of the element, and hence can be used for subsequent focussing of the atomic beam. This focussing is analogous to the focussing of light through a conventional thin lens.

Our design is very cost effective and easily adaptable to any atomic beam line. Due to the design of the slowing system, it has the significant benefit of being mounted inline with the atomic beam line thereby maximizing the transmission of atoms through the guide. It would be easy to adapt so that the device could act in a dual role as a conductance aperture for differentially pumped UHV vacuum systems such as those used in Bose Einstein condensation experiments.

The magnetic guide is constructed of nine individual magnetic hexapole lens [21] elements, arranged such that the initial and final two elements are axially aligned while the inner five elements are aligned on a 30 degree arc with a radius of curvature of $30 \mathrm{~cm}$, as shown in Figure 2a. Each hexapole lens element consists of twelve permanent rare earth magnets held in formation by an aluminium housing with a central aperture of $23.5 \mathrm{~mm}$, as in Figure $2 \mathrm{~b}$. 
The dimensions of the magnets used are only $6.4 \times 6.4 \times 12.7 \mathrm{~mm}$ and have a surface field strength of $\sim 0.44 \mathrm{~T}[22]$. The entire lens system is mounted within a custom fabricated 304 stainless steel chamber with 6" CFF fittings so that it can be mounted in-line with the remainder of the atomic beam line.

The force that an atom with a magnetic moment feels in the presence of a magnetic field gradient is given by

$$
\boldsymbol{F}=\mu_{B} g_{j} m_{j} \nabla \boldsymbol{B}
$$

where $\mu_{B}$ is the Bohr magneton, $g_{j}$ is the Landé $\mathrm{g}$ factor, and $m_{j}$ is the magnetic substate. The spacial variation of an ideal hexapole magnetic field is given by [23]

$$
|\boldsymbol{B}(r)|=B_{r}\left(\frac{r}{r_{0}}\right)^{2}
$$

where $B_{r}=1.32 \mathrm{~T}$ is the remanent field, $r$ is the distance from the center of the magnetic hexapole element, and $r_{0}$ is the distance from the inner boundary of the individual magnets to the center. This field profile was assumed to be constant over the short length of the hexapole lens in our initial simulations. With this information a two-dimensional MonteCarlo simulation of the atom trajectories through the magnetic guide was carried out using Matlab, enabling the design parameters to be determined. In our design, the magnets, with dimensions and magnetic field strengths mentioned previously, were arranged such that the smallest internal radius was achieved. Therefore the design parameters that could be modified for optimal operation were the longitudinal velocities of the incident atoms and the radius of curvature of the magnetic guide. Additional simulations can also be carried out to optimize the arrangement and dimensions of the each magnetic lens element. This was not performed here as inexpensive, commercially available magnets prove to be more than sufficient to guide metastable neon atoms in the $m_{j}=+2$ magnetic substate. Once the hexapole magnetic lens elements were constructed the magnetic field profile could be further investigated.

The spacial variation in the magnetic flux density for a hexapole magnet, such as our design, has been modelled by Halbach [23]. However, in our design the individual permanent magnets have a rectangular cross section whereas the theory of Halbach is for magnets with a trapezoidal cross section [23]. As such, this theory cannot be used to directly describe 


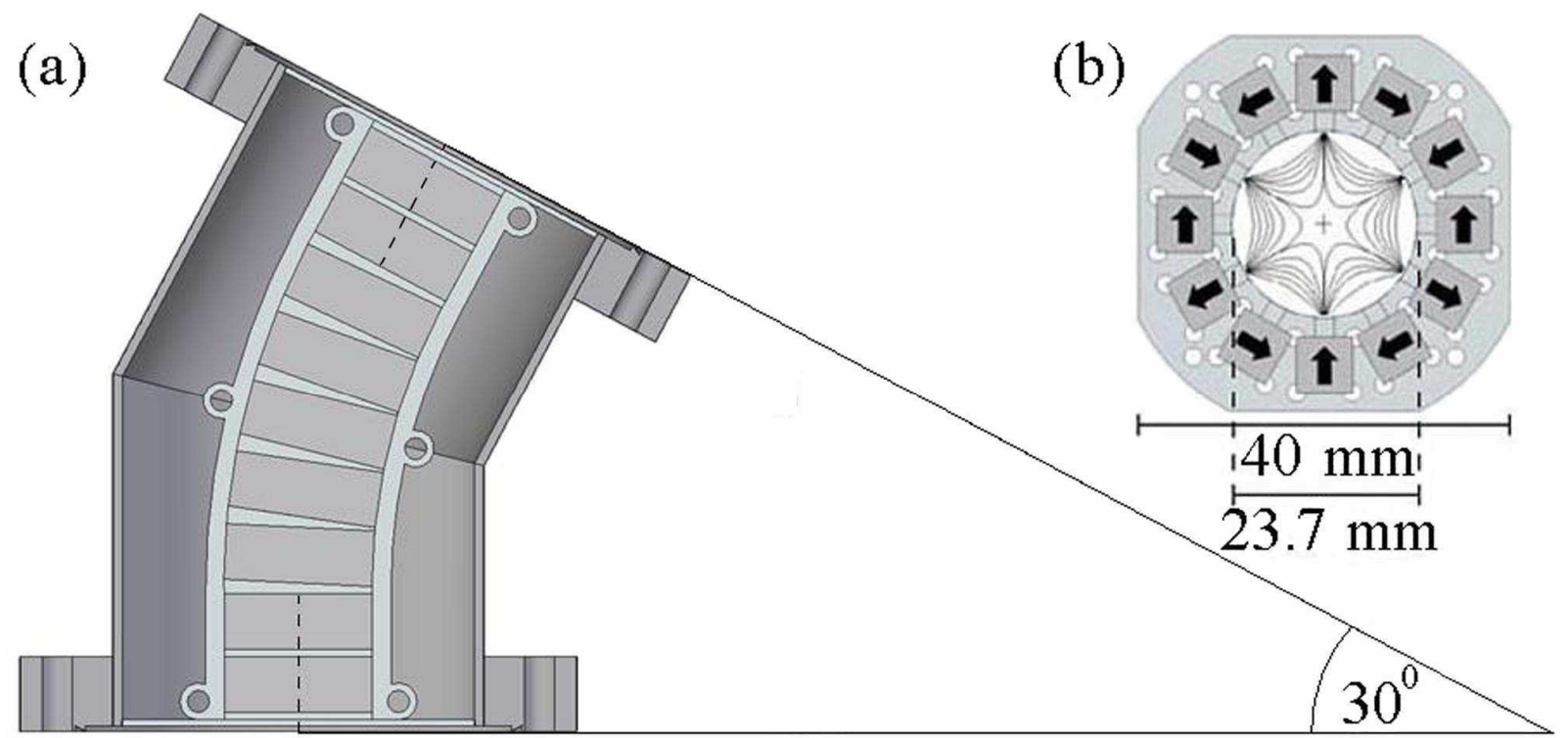

\section{$300 \mathrm{~mm}$}

FIG. 2: a) The nine element magnetic guide arrangement, b) single hexapole lens element showing magnetic field contour lines.

the magnetic flux density within this lens. The magnetic field at the central point of the hexapole lens elements was profiled using a Hall probe by obtaining line profiles over multiple angles between 0 and 60 degrees (where the angle is defined in Figure 3a). Profiles at larger angles were not required due to the symmetry of the magnetic field. This data enabled a two-dimensional magnetic field profile to be generated by a Matlab program that produced the output magnetic flux density profile as shown in (Figure 3a). The figure shows that beyond approximately $10 \mathrm{~mm}$ from the center of the lens, the uniformity of the magnetic field gradient is lost due to the geometry of the magnets used. It also shows that the theory predicts the magnetic flux density to within $1 \%$ until $\sim 11 \mathrm{~mm}$ from the center of each magnetic lens element, which is close to the edge of the lens. As mentioned previously, the theory of Halbach [23] could not be used to directly determine the magnetic flux density, however, our work shows that the changes in magnetic flux density with respect to the angle defined in (Figure 3a) can be reasonably well described by including correction terms [24] taking into account the segmentation of the multipole magnetic field derived by Halbach. In Figure 3b three measured line profiles of the magnetic field, defined by the angle with respect to the $\mathrm{x}$-axis of Figure 3a, are plotted and show good comparison to the theory of Halbach (with correction terms). 


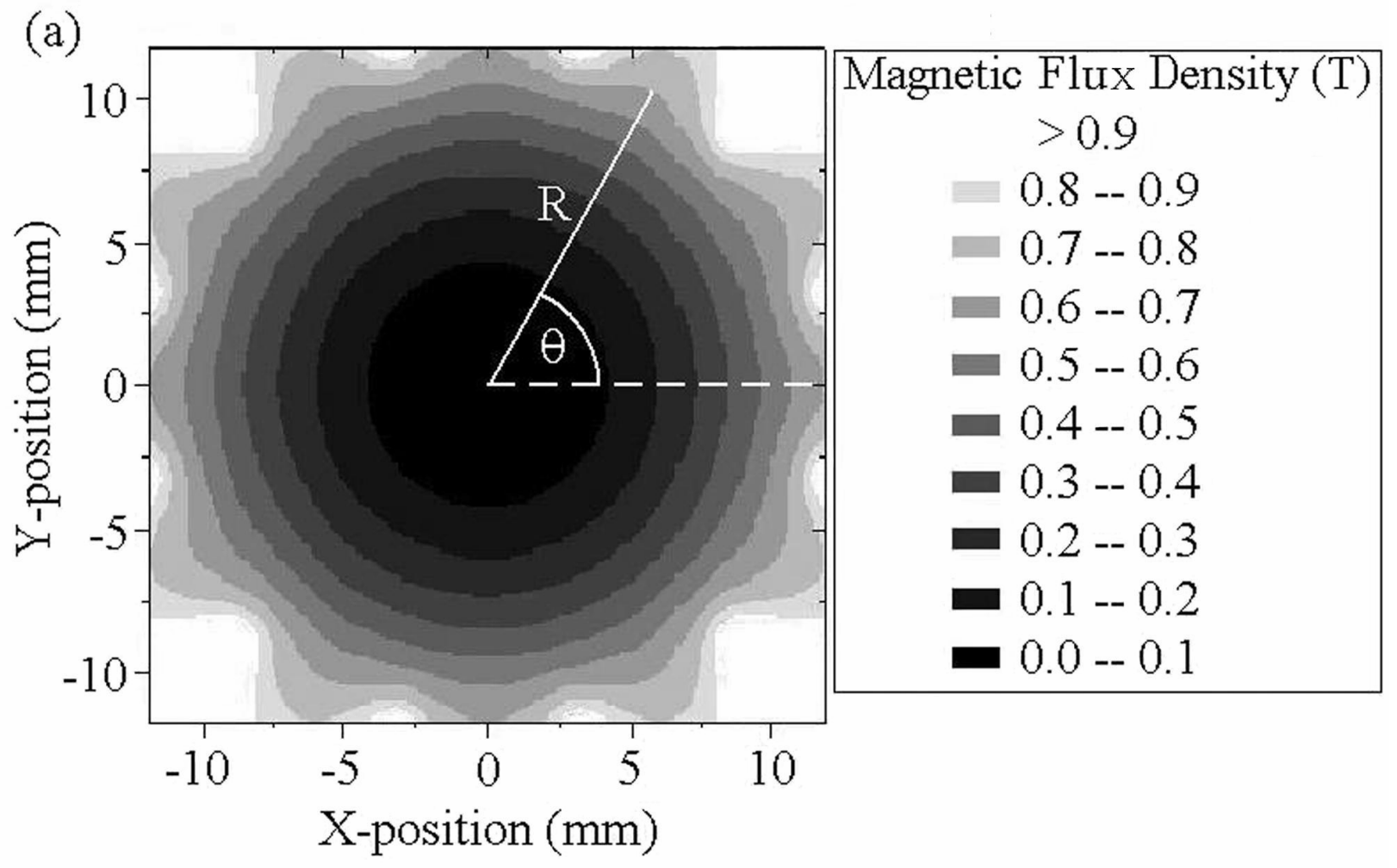

(b)

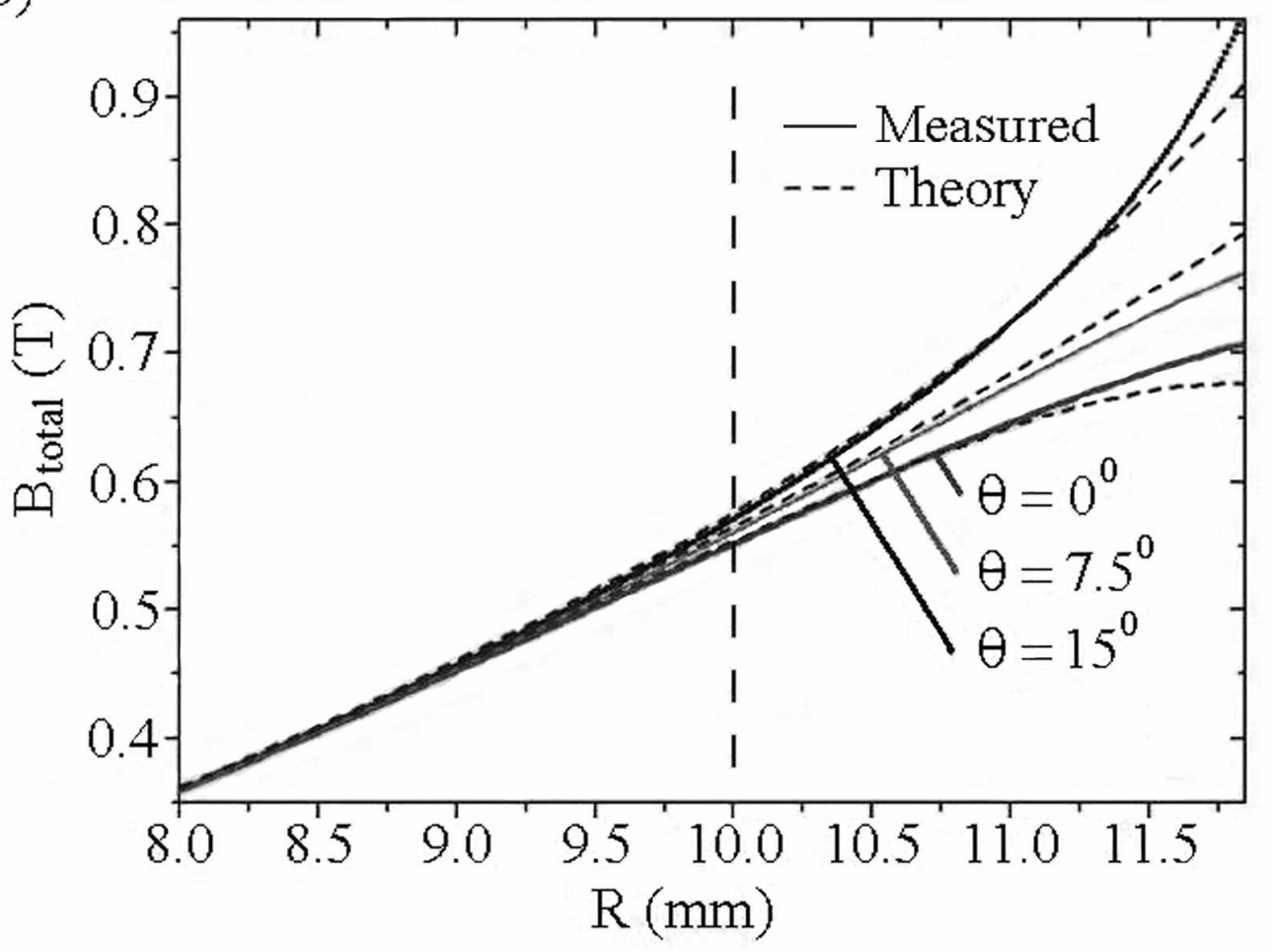

FIG. 3: a) Measured magnetic field profile of the lens in figure 2b, b) plot displaying the angular dependence of the magnetic field as measured and as predicted by theory [23], with the point at which the magnetic flux density differs by $\sim 1 \%$ indicated by the vertical dashed line. 
The magnetic field inside the hexapole lens was then profiled in the xz-plane relative to Figure 3a, such that subsequent simulations could include an accurate three dimensional description of the magnetic flux density.

These simulations show that atoms in the $m_{j}=+2$ magnetic substate travelling with a longitudinal velocity of $80 \mathrm{~m} \mathrm{~s}^{-1}$ and a maximum transverse velocity of $\pm 4 \mathrm{~m} \mathrm{~s}^{-1}$ are guided through the 30 degree arc. These conditions correspond to the most probable velocity of the atoms exiting the Zeeman-slower and the degree of collimation of the atomic beam. A significant proportion of the atoms in the $m_{j}=+1$ magnetic substate also successfully emerge from the magnetic guide under the same initial conditions. Figure 4 shows the transmission efficiency of both the $m_{j}=+1$ and +2 substates relative to the initial position of the atoms as they enter the guide. While this simulation was only carried out for an atomic beam with our experimental parameters, the effect of increasing the transverse velocities, and hence input angle, of the atoms entering the magnetic guide was to significantly decrease the transmission. Initial estimates showed that by increasing the divergence of the incident atomic beam by a factor of two, decreased the transmitted flux by nearly half. The aperture area exposed to atoms under experimental conditions is represented by the rectangle in the figure. In order to reduce the complexity of the simulations the approximation was made that the lens elements were evenly spaced around the 30 degree arc of the magnetic guide. This is not the case due to the geometric restrictions of the lens elements around the bend as shown in Figure 2a. While the simulations do not exactly correspond to the experimental conditions as a result of these slight geometric variations, an approximate expectation value of the transmission efficiency can be obtained. From the investigation of the transmission through the central seven magnetic lens elements the transmission efficiency should be within $\pm 5 \%$ of the experimental value.

\section{RESULTS}

The total atom flux of the source measured $395 \mathrm{~mm}$ downstream from the optical collimator was $1.60 \times 10^{12} \pm 4 \times 10^{10}$ atoms s$^{-1}$. After longitudinal slowing the total slowed atom flux was $5.2 \times 10^{10} \pm 1 \times 10^{9}$ atoms s$^{-1}$, which upon comparison to the unslowed atom flux results in a slowing efficiency of $\sim 58 \%$. The atomic flux is reduced by two orders of magnitude due to the appreciable divergence of the atomic beam over the large path length. 
(a)

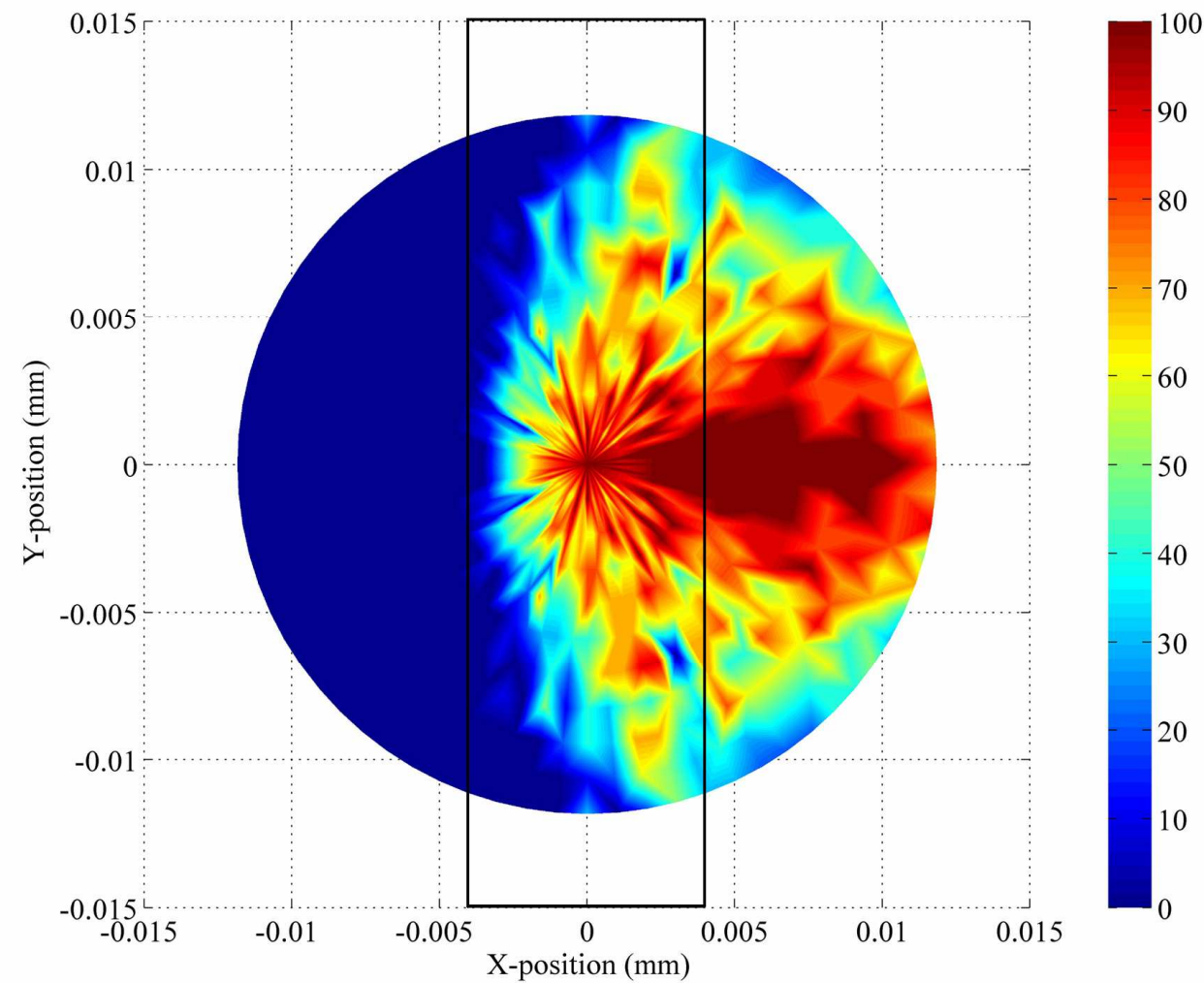

(b)

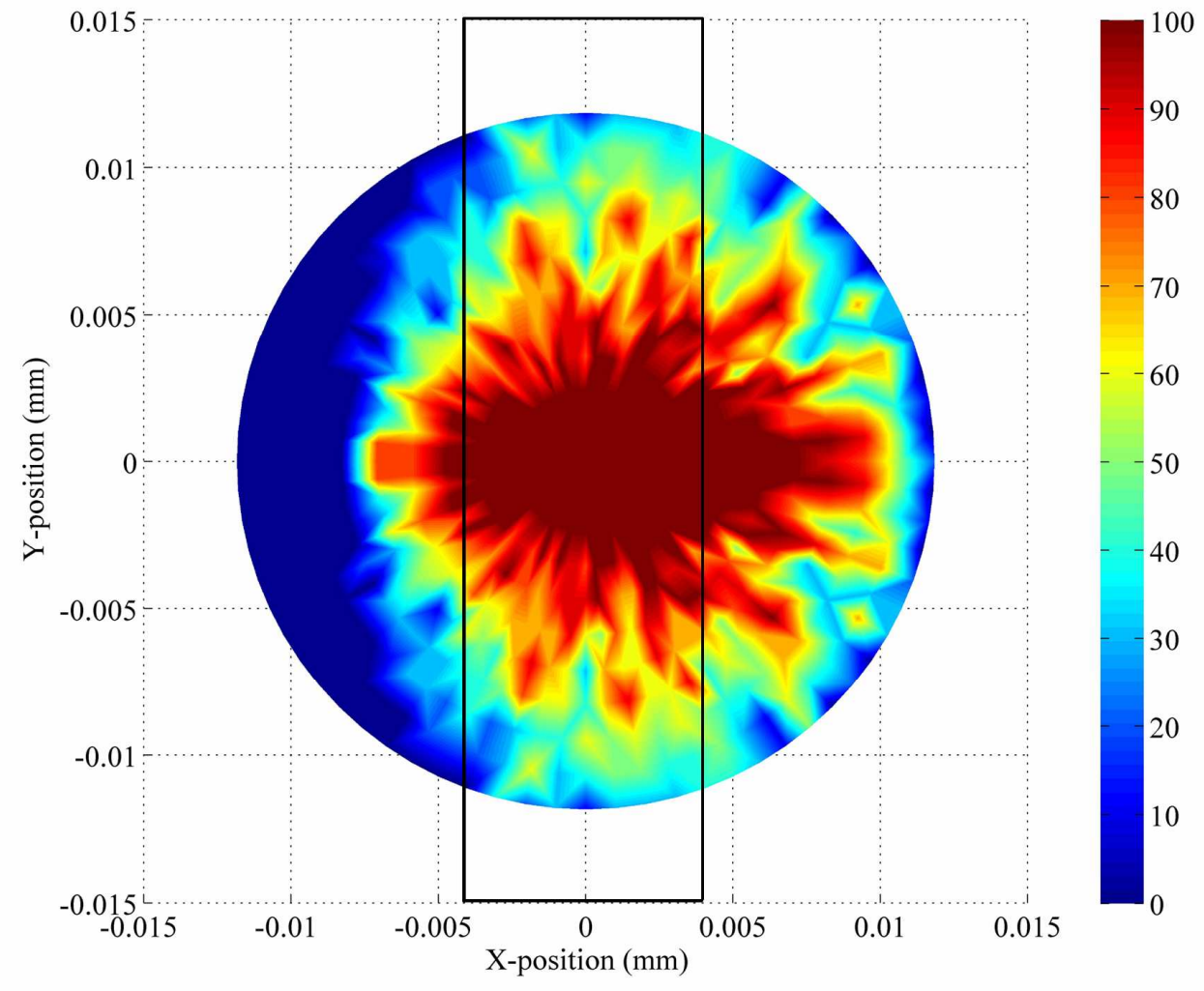

FIG. 4: (Color online) a) Transmission efficiency, relative to initial positions, of atoms in the $m_{j}=+1$ substate, b) transmission efficiencies for atoms in the $m_{j}=+2$ substate. 
The atomic beam incident on the magnetic guide consists largely of atoms in the ${ }^{3} \mathrm{P}_{2}$ and ground states with less than $2 \%$ of the total flux in the ${ }^{3} \mathrm{P}_{0}$ metastable state and only $\sim 0.7 \%$ UV photons. Once the atomic beam is guided through a 30 degree angle the flux is reduced further to $4.35 \times 10^{9} \pm 2 \times 10^{7}$ atoms $^{-1}$, giving a transmission efficiency of the magnetic guide of $\sim 9 \%$. However the emergent beam is purely atoms in the ${ }^{3} \mathrm{P}_{2}$ metastable state as all other constituents are not guided through the 30 degree angle. The measured value for transmission efficiency is somewhat lower than that predicted by simulations of the magnetic guide, $\sim 23 \%$. There are two possible contributions to this discrepancy. Firstly, it was assumed that each magnetic substate was equally populated upon the entrance to the magnetic guide. The different magnetic substates are guided with differing efficiencies, as the nature of the guiding force is dependant upon the magnetic substate. Hence, if the $m_{j}=+2$ magnetic substate is less populated than assumed, the measured efficiency will decrease. This would be the case if the polarization of the recollimation optical molasses was not properly defined. In this experiment the recollimation beams are linearly polarized which has the effect of optically pumping the atoms into magnetic substates with low magnetic projection quantum numbers. Also, the slight differences in the simulated and experimental magnetic guide arrangement will have some small but finite (expected to be $\approx 5 \%$ ) contribution to the discrepancy observed.

It should be noted that although this magnetic guide was constructed for a 30 degree deviation of the atomic beam, deviations of much larger angles can be realized. Confirmation that the beam was free of UV photons was carried out via the introduction of a buffer gas to collisionally de-excite the metastable atoms.

Previous atom lithography experiments utilized an exposure time on the order of one hour $[8,25]$. Assuming the flux measurement is for an atomic beam the size of the guide aperture, and knowing the dosage required for the formation of patterning in a dodecanethiol (DDT) resist coated sample, the calculated exposure time required after the magnetic guide is $>40$ hours which is impractical. However, the total flux achieved after the magnetic guide is significantly larger in comparison to similar apparatus [10], and the beam brightness can be significantly improved by focussing the atomic beam or via the implementation of a subsequent magneto-optic compression element [26]. Such elements should readily be able to provide larger than $10^{2}$ times flux enhancement of the atomic beam which could result in an exposure time of $\sim 30$ minutes for this experiment. 


\section{Acknowledgments}

This work was supported by Griffith University and the Australian Research Council. J.P. and A.J. were supported by the Australian Research Council.

[1] W. Gerlach and O. Stern, Z. Phys. (1922).

[2] H. Freidberg and W. Paul, Naturwiss 38, 159 (1951).

[3] C. Adams, M. Sigel, and J. Mlynek, Phys. Reports 240, 143 (1994).

[4] H. J. Metcalf and P. van der Straten, Laser Cooling and Trapping (Springer-Verlag, New York, 1999), chapter 15.

[5] B. Rohwedder, Am. J. Phys. 75, 394 (2007).

[6] K. K. Berggren, A. Bard, J. L. Wilbur, J. D. Gillaspy, A. G. Helg, J. J. McClelland, S. L. Rolston, W. D. Phillips, M. Prentiss, and G. M. Whitesides, Science 269, 1255 (1995).

[7] T. Gay, Exp. Methods Phys. Sci. B 29, 95 (1996).

[8] M. Baker, A. J. Palmer, W. R. MacGillivray, and R. T. Sang, Nanotechnology 15, 1356 (2004).

[9] J. E. Bjorkholm, R. R. Freeman, and D. B. Pearson, Phys. Rev. A 23, 491 (1981).

[10] A. Scholz, M. Christ, D. Doll, J. Ludwig, and W. Ertmer, Opt. Commun. 111, 155 (1994).

[11] R. R. Chautowski, V. Y. F. Leung, and K. G. H. Baldwin, Appl. Phys. B 86, 491 (2007).

[12] M. Baker, A. J. Palmer, and R. T. Sang, Meas. Sci. Technol. 14, N5 (2003).

[13] A. J. Palmer, M. Baker, and R. T. Sang, Rev. Sci. Inst. 75, 5056 (2004).

[14] J. P. Ashmore and R. T. Sang, Meas. Sci. Technol. 12, N17 (2001).

[15] M. D. Hoogerland, J. P. J. Driessen, E. J. D. Vredenbregt, H. J. L. Megens, M. P. Schuwer, H. C. W. Beijerinck, and K. A. H. van Leeuwen, Appl. Phys. B 62, 323 (1996).

[16] F. Shimizu, K. Shimizu, and H. Takuma, Phys. Rev. A 39, 2758 (1989).

[17] H. J. Metcalf and P. van der Straten, Laser Cooling and Trapping (Springer-Verlag, New York, 1999), appendix C.

[18] S. Schohl, D. Klar, T. Kraft, H. A. J. Meijer, M. W. Ruf, U. Schmitz, S. J. Smith, and H. Hotop, Z. Phys. D 21, 25 (1991).

[19] A. Goepfert, F. Lison, R. Schütze, R. Wynands, D. Haubrich, and D. Meschede, Appl. Phys. B 69, 217 (1999). 
[20] B. Ghaffari, J. M. Gerton, W. I. McAlexander, K. E. Strecker, D. M. Homan, and R. G. Hulet, Phys. Rev. A 60, 3878 (1999).

[21] W. G. Kaenders, F. Lison, I. Müller, A. Richter, R. Wynands, and D. Meschede, Phys. Rev. A 54, 5067 (1996).

[22] Magnets were sourced from K\&J magnetics inc., Part No. B842.

[23] K. Halbach, Nucl. Instrum. Methods 169, 1 (1980).

[24] K. C. Kuiper, Master's thesis, Eindhoven University of Technology, 2007.

[25] A. J. Palmer, M. Baker, and R. T. Sang, Nanotechnology 17, 1166 (2006).

[26] J. Nellessen, J. Werner, and W. Ertmer, Opt. Commun. 78, 300 (1990). 